\title{
Use of Computerized Tomography and Chest X-Rays in Evaluating Efficacy of Aerosolized Recombinant Human DNase in Cystic Fibrosis Patients Younger Than Age 5 Years: A Preliminary Study
}

\author{
Samya Z. Nasr, MD, ${ }^{*}$ Lawrence R. Kuhns, MD, Randall W. Brown, MD, Martin E. Hurwitz, MD, \\ Georgiana M. Sanders, MD, and Peter J. Strouse, MD
}

\begin{abstract}
Summary. The aim of this study was to evaluate the ability of high-resolution computerized tomography (HRCT) of the chest and chest x-rays (CXR) to determine efficacy of inhaled recombinant human DNase (rhDNase) in cystic fibrosis (CF) patients younger than 5 years of age. A randomized, double-blind, placebo-controlled pilot study of 12 patients with CF younger than 5 years of age, attending the University of Michigan Cystic Fibrosis Center (Ann Arbor, MI) was conducted. The changes in the HRCT and CXR score from baseline to day 100 of therapy were assessed using a previously validated scoring system.

The mean changes of HRCT scores between the rhDNase and placebo groups were found to be significant at the $95 \%$ level, with mean change \pm SE mean of $-1.00 \pm 0.53$ and $0.58 \pm 0.24$ for rhDNase and placebo groups, respectively $(P=0.02)$. The difference in CXR score was not significant between the two groups. An analysis was performed to relate HRCT subscores to CXR score; only thickening of the intra-interlobular septae was significantly correlated with the total CXR score $(r=-0.7, P<0.01)$. There was improvement in the parents' assessments of the patients' well-being, with improvement in physical activity, decreased cough, sleep quality, and appetite in those subjects receiving rhDNase.

We conclude that the administration of rhDNase was associated with improvement in the HRCT scan in CF patients younger than 5 years of age. Findings indicate that HRCT of the chest is useful and sensitive in studying responses to therapy in patients with CF lung disease. To our knowledge, this is the first report of the use of HRCT to assess the effectiveness of a therapeutic modality in so young a CF patient population. Pediatr Pulmonol. 2001; 31:377-382.

๑) 2001 Wiley-Liss, Inc.
\end{abstract}

Key words: preschool children; cystic fibrosis; high-resolution computed chest tomography; recombinant human DNase; pulmonary disease.

\section{INTRODUCTION}

Respiratory disease in patients with cystic fibrosis is caused by the accumulation of purulent, infected sputum in the airways and leads to obstruction of the small airways, and a state of chronic low-grade infection. ${ }^{1}$ Sputum in CF is rich in leukocyte-derived DNA that greatly contributes to abnormal viscoelasticity of the $\mathrm{CF}$ sputum. ${ }^{2}$ CF patients younger than 5 years of age have significant amounts of DNA in their airway. ${ }^{3}$ Recombinant human DNase 1 (rhDNase), a copy of the native human enzyme responsible for hydrolyzing extracellular DNA, dramatically alters the viscoelastic properties of $\mathrm{CF}$ sputum in vitro. ${ }^{4}$ Aerosol administration of rhDNase (c) 2001 Wiley-Liss, Inc.
Sections of Pediatric Pulmonary and Radiology, Department of Pediatrics, University of Michigan Medical Center, Ann Arbor, Michigan.

Grant sponsor: NIH; Grant number: MO1-RR000042; Grant sponsor: Genentech, Inc.; Grant sponsor: Dale Maxwell Memorial Funds.

Presented in part at the International Conference for the American Thoracic Society, April 23-28, 1999, and at the North American Annual Cystic Fibrosis Conference, October 7-10, 1999.

*Correspondence to: Samya Z. Nasr, M.D., Department of Pediatrics, University of Michigan Medical Center, 1500 E. Medical Center Drive, Ann Arbor, MI 48109-0212. E-mail: snasr@umich.edu

Received 29 March 2000; Accepted 12 January 2001. 
(Pulmozyme ${ }^{\circledR}$, Genentech, Inc., South San Francisco CA) with a pneumatically driven hand-held nebulizer improves pulmonary function in CF patients. The improvement was noted within days of initiating therapy. ${ }^{5,6}$ In addition, long-term administration of rhDNase reduced the need for parenteral antibiotic treatment of respiratory tract infections and the rate of hospitalization. ${ }^{7}$ Most efficacy studies have been done in CF patients older than 5 years of age because they can perform pulmonary function testing to evaluate objectively the efficacy of this drug. The safety of rhDNase has been studied in CF children younger than 5 years of age. ${ }^{8}$ Patients younger than 5 years of age have been excluded from efficacy trials because of their inability to perform pulmonary function tests with predetermined standards. However, $17.6 \%$ of the CF patient population in the USA in 1997 were younger than 5 years of age; of these, 598 patients $(16.2 \%$ of the total in that age group) were reported to be receiving rhDNase. ${ }^{9}$

High-resolution computed tomography (HRCT) of the chest has been used to evaluate the progression and severity of lung disease in $\mathrm{CF}^{10-14}$ Several scoring systems for HRCT have been developed to evaluate lung disease in CF. ${ }^{11,12}$ Multiple studies have shown that CT scanning is more sensitive and specific than chest radiography. ${ }^{11,12,14,15} \mathrm{CT}$ scanning can localize and qualitatively describe focal areas of parenchymal abnormality. ${ }^{16}$

We designed a randomized, double-blind, placebocontrolled clinical trial to evaluate the ability of HRCT and chest x-rays (CXR) to detect lung changes after administering rhDNase in children younger than 5 years of age with $\mathrm{CF}$.

\section{PATIENTS AND METHODS}

Patients with $\mathrm{CF}$ younger than 5 years of age and attending the University of Michigan CF Center were considered eligible for the study. We enrolled 12 patients (8 males and 4 females) with a mean age of $3.06 \pm$ 1.49 years (mean \pm SD), and a range of $1.10-4.99$ years, all with a confirmed diagnosis of CF by abnormal values of sweat chloride (Table 1). To ensure that patients were clinically stable prior to enrollment, patients receiving antibiotics within 2 months prior to enrollment and who were hospitalized for a pulmonary exacerbation within 3 months prior to enrollment were excluded. No participants had received rhDNase or any investigational drug

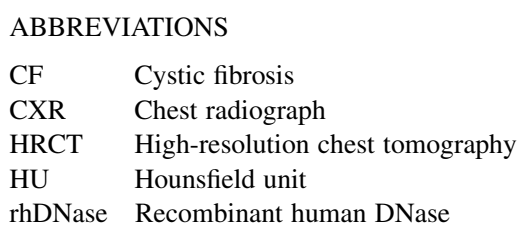

TABLE 1-Characteristics of Study Population at Baseline

\begin{tabular}{lcc}
\hline Characteristics & Placebo & rhDNase \\
\hline 1) Number of patients & 6 & 6 \\
2) Age (years) & $2.64 \pm 1.64^{1}$ & $3.48 \pm 1.32^{1}$ \\
& $($ range; $1.10-4.99)$ & (range; $1.65-4.70)$ \\
3) Gender (M/F) & $4 / 2$ & $4 / 2$ \\
4) Sweat chloride (meq/L) & $99.33 \pm 12.82^{1}$ & $104.60 \pm 9.07^{1}$ \\
(range; $90-125)$ & $($ range; $94-119)$ \\
5) Genetic analysis & 3 & 2 \\
$\quad$ Homozygous delta F508 & 2 & 3 \\
$\quad$ Heterozygous delta F508 & 6 & 3 \\
6) Airway colonization & & \\
with Pseudomonas & & 5 \\
$\quad$ aeruginosa (no.) & 5 & 6 \\
7) Chest physiotherapy & 6 & \\
8) Bronchodilators & & \\
\hline
\end{tabular}

${ }^{1}$ Mean \pm SD.

therapy within 6 months prior to enrollment. All patients had received standard care for cystic fibrosis. ${ }^{17}$ Patients were assigned at random to one of the study groups.

Patients were randomly assigned to receive either $2.5 \mathrm{mg}$ of rhDNase or placebo once a day for 100 days. The dose was based on the results of a previous study. ${ }^{7}$ Both physicians and parents were blind with respect to the treated and placebo groups. Genentech, Inc. provided the rhDNase (Pulmozyme ${ }^{\mathbb{R}}$ ) and placebo used in the study. rhDNase was provided as a solution $(1 \mathrm{mg} / \mathrm{mL})$ in $2.5 \mathrm{~mL}$ of excipient $(150 \mathrm{mM}$ sodium chloride, $1.5 \mathrm{mM}$ calcium chloride, $\mathrm{pH}$ 6.0). The placebo was excipient alone. The study drug was nebulized once a day using the ProNeb ${ }^{\mathbb{R}}$ compressor/nebulizer system (PARI Respiratory Equipment, Inc., Midlothian, VA). Vials were returned and counted at the end of the study as a measure of compliance. The study was reviewed and approved by the University of Michigan Institutional Review Board, and informed consent was obtained from all parents or guardians.

The patients were evaluated upon enrollment and at the end of the study. Blinding for the study was done in a random fashion, regardless of the number of pulmonary exacerbations or colonization status prior to enrollment. Anteroposterior and lateral CXR projections and HRCT were obtained on both occasions. A questionnaire was developed and used to assess the patients' general sense of well-being (with questions about activity level, appetite, and sleep pattern) and CF-related symptoms (with questions about frequency and nature of cough, wheezing, retraction, and shortness of breath). The parents completed a questionnaire upon enrollment and at the end of the study. The number of days of daycare or preschool missed because of $\mathrm{CF}$ related illnesses were recorded. Upon enrollment, a deep oropharyngeal culture was obtained to assess colonization status with Pseudomonas aeruginosa (Table 1). 
HRCT scan was done using 40 milliamperes, $1 \mathrm{sec}$ scan time per slice, and $140 \mathrm{kV}$ for each of five evenly spaced 1-mm-thick slices through the lungs. The CT scanner was a HiSpeed Advantage ${ }^{\circledR}$ scanner (General Electric Medical Systems, Milwaukee, WI). Because of the speed of the scanner and the limited number of slices (five), HRCT was done without sedation. Based on the study of Mayo et al. ${ }^{16}$ and phantom (plastic model simulating cross section of the lung) studies done on our scanner,${ }^{19}$ we estimated the peak dose within each slice to be 300-500 mrem and the average dose to the entire lung to be $50 \mathrm{mrem}$. Each slice was processed using a bone algorithm and photographed 4 on 1 on a 14 inch by 17 inch film, using a window width of 1,000 Hounsfield units (HU) and a level of $-600 \mathrm{HU}$.

The HRCT scans were scored by two pediatric radiologists (P.J.S. and K.A. Garver), using the modified HRCT scoring system described by Santamaria et al., ${ }^{20}$ modified from Bhalla et al. ${ }^{11}$ Both radiologists were blind to the patients' treatment assignment. HRCTs were read at random. The HRCT final score of each patient was calculated on the basis of severity and extent of the morphologic changes, and represented the average score from two observers. The highest possible score was 30, which indicated severe lung disease.

The chest radiographs were scored independently and in a blinded manner by a pediatric radiologist (L.R.K.) and a pediatric pulmonologist (S.Z.N.) using the Brasfield score, ${ }^{21}$ and the scores were averaged. This scoring system has been used to evaluate the severity of CF lung disease. A score of 25 points indicates normal lungs. The score decreases with increasing severity of lung disease.

The CXR and HRCT images were presented in random, independent order for evaluation by two observers. To estimate the interobserver reliability in scoring CXR and HRCT images, the interobserver coefficients of variation of either CXR or HRCT score were calculated using the formula:

Coefficient of variation

$$
=\frac{\begin{array}{c}
\text { Absolute mean difference } \\
\times 100(\text { expressed as a percentage })
\end{array}}{\text { Overall mean of scores }}
$$

where the "absolute mean difference" is the mean of the absolute value in the differences in the scores of the two observers, and the "overall mean of scores" is the mean of the scores of the two observers for all subjects included. Differences in means were tested for significance in assessing interobserver reliability, using a paired samples $t$-test. Correlation coefficients were tested for significance using a Pearson's product-momentum coefficient.

Comparative analyses of the two groups in the clinical trial are presented as mean $\pm \mathrm{SD}$. Differences in means were tested in these cases, using a $t$-test for independent samples. This was a pilot study; no sample size calculation was performed. The number of subjects studied was arbitrarily chosen. All analyses were carried out in SPSS (Statistical Package for Social Science) (SPSS, Inc., Chicago, IL)

\section{RESULTS}

Twelve patients were enrolled and randomized to one of two treatment groups. Clinical characteristics of the two groups are presented in Table 1. One patient in the rhDNase group used only two vials; the rest of the patients in the two groups used 89-100 vials. Most of the study population had mild respiratory disease as assessed by CXR score ( 10 patients scored $\geq 21,1$ patient scored 20, and 1 patient scored 18 Brasfield units) and HRCT score (11 patients scored $<5$ and 1 patient scored 8.5). There was no difference in CXR score at baseline between the two groups.

Table 2 represents a summary of HRCT scores findings in the two study groups. The HRCT scans were free of respiratory motion artifact in 5 out of 6 patients older than 2.2 years, and in 1 out of 6 patients younger than 2.2 years. Motion artifact assessment was subjective. The two readers were able to read and score all HRCTs. Table 3 summarizes the means $\pm \mathrm{SD}$ and ranges for CXR and HRCT scores for both study groups before and at the end of the study period.

The CXR scoring system had excellent interobserver reliability. The interobserver coefficient of variation for the CXR score was $3.1 \%$. The HRCT score had a higher interobserver coefficient of variation (49.1\%). That was due to the low scoring scale for HRCT scans (10 points maximum) vs. higher scoring scale for CXR (25 points), and the small number of subjects in this study. Mean CXR scores showed a trend towards a significant statistical difference between the two observers ( $22.17 \pm 0.61$ vs. $21.50 \pm 0.51$ (mean \pm SEM); $P=0.09$ ). There was no statistically significant difference between the two observers for mean HRCT scores $(2.00 \pm 0.83$ vs. $2.42 \pm 0.66 ; P=0.38)$. The correlation coefficients for CXR and HRCT scores between the two observers were 0.82 and 0.84 , respectively, with both values being statistically significant $(P<0.001)$. There was no statistically significant correlation between CXR and HRCT scores $(\mathrm{r}=-0.4, P=0.15)$.

There was a significant correlation between specific HRCT changes for bronchiectasis, peribronchial thickening, mucus plugging, and air trapping, and the total HRCT score $(\mathrm{r}=0.8, \mathrm{r}=0.9, \mathrm{r}=0.8, \mathrm{r}=0.9$ respectively, $P<0.001$ for all subscores). Acinar nodules/consolidation, thickening of intra-interlobular septae, and ground glass opacities were significantly correlated to the total HRCT score, with $P<0.05$ and $\mathrm{r}=0.6$ for all subscores. When the analysis was performed to relate the HRCT 
TABLE 2-Summary of HRCT Findings in Study ${ }^{1}$

\begin{tabular}{|c|c|c|c|c|}
\hline \multirow[b]{2}{*}{ Category } & \multicolumn{2}{|c|}{ Placebo } & \multicolumn{2}{|c|}{ rhDNase } \\
\hline & Before & After & Before & After \\
\hline Severity of bronchiectasis & $0.08 \pm 0.29$ & $0.25 \pm 0.45$ & $0.17 \pm 0.39$ & $0.08 \pm 0.29$ \\
\hline Peribronchial thickening & $0.42 \pm 0.51$ & $0.58 \pm 0.67$ & $0.58 \pm 0.67$ & $0.38 \pm 0.48$ \\
\hline Extent of bronchiectasis & $0.08 \pm 0.29$ & $0.25 \pm 0.45$ & $0.17 \pm 0.39$ & $0.08 \pm 0.29$ \\
\hline Extent of mucus plugging & $0.00 \pm 0.00$ & $0.08 \pm 0.29$ & $0.17 \pm 0.39$ & $0.17 \pm 0.39$ \\
\hline Number of bullae & $0.00 \pm 0.00$ & $0.00 \pm 0.00$ & $0.00 \pm 0.00$ & $0.00 \pm 0.00$ \\
\hline Air trapping & $0.42 \pm 0.67$ & $0.25 \pm 0.45$ & $0.50 \pm 0.67$ & $0.08 \pm 0.29$ \\
\hline Collapse (atelectasis) & $0.00 \pm 0.00$ & $0.00 \pm 0.00$ & $0.00 \pm 0.00$ & $0.00 \pm 0.00$ \\
\hline Acinar nodules/consolidations & $0.17 \pm 0.39$ & $0.42 \pm 0.51$ & $0.17 \pm 0.39$ & $0.17 \pm 0.39$ \\
\hline Thickening of intra-interlobular septae & $0.33 \pm 0.49$ & $0.25 \pm 0.45$ & $0.08 \pm 0.29$ & $0.00 \pm 0.00$ \\
\hline Ground-glass opacities & $0.58 \pm 0.67$ & $0.58 \pm 0.67$ & $0.50 \pm 0.67$ & $0.33 \pm 0.49$ \\
\hline
\end{tabular}

${ }^{1}$ Mean \pm SD. For all categories, a score of 0.00 indicates an absence of changes, and a score of 3 indicates severe changes.

TABLE 3-Chest Radiograph and HRCT Comparison ${ }^{1}$

\begin{tabular}{lrrrrr}
\hline Variables & \multicolumn{2}{c}{ Placebo } & \multicolumn{2}{c}{ rhDNase } \\
\hline Chest radiograph score & & & & \\
$\quad$ Before & $21.83 \pm 2.32$ & $(18-25)$ & $21.84 \pm 1.60$ & $(20-23.5)$ \\
$\quad$ After & $20.58 \pm 2.99$ & $(16.5-24.5)$ & $22.50 \pm 1.50$ & $(20.5-24)$ \\
HRCT & & & & & \\
$\quad$ Before & $2.08 \pm 1.93$ & $(0-5)$ & $2.33 \pm 2.02$ & $(0-10)$ \\
$\quad$ After & $2.67 \pm 2.49$ & $(0-7)$ & $1.33 \pm 2.03$ & $(0-6)$ \\
\hline
\end{tabular}

${ }^{1}$ Plus-minus values, mean \pm SD. Ranges are in parentheses.

subscores to CXR score, only thickening of the intrainterlobular septae was significantly correlated with the total CXR score $(\mathrm{r}=-0.7, P<0.01)$.

We compared the effects of rhDNase treatment and the placebo group, using the CXR and HRCT scores. There was no significant difference between either the CXR scores or the HRCT scores between the two groups at baseline. To compare the effects of the treatment on the two groups, a "gain score" was calculated by subtracting the CXR and HRCT scores before treatment from the scores after treatment. When the change in HRCT scores over the treatment period was calculated, the difference in the mean change between the rhDNase and placebo groups was found to be significant, with mean changes \pm SE being $-1.00 \pm 0.53$ and $0.58 \pm 0.24$ for the rhDNase and placebo groups, respectively $(P=0.02)$. The difference in the mean change in CXR scores was not found to be statistically significant, with mean $\pm \mathrm{SE}$ being $0.67 \pm 0.81$ and $-1.25 \pm 0.97$ for the rhDNase and placebo groups, respectively $(P=0.16)$.

Parents of 4 of the 6 patients randomized to rhDNase recorded improvement in patients' physical activity, decreased cough, better sleep quality, and improved appetite. Parents of 4 of the 6 patients in the placebo group recorded lack of improvement while in the study. Parents of 2 of the 6 patients in each group were not sure if their children's clinical status changed during the study period. No significant adverse effects were attributed to the administration of rhDNase.

\section{DISCUSSION}

In this preliminary, short-term study, we demonstrated the efficacy of aerosolized rhDNase in patients younger than 5 years of age with cystic fibrosis. Since standards have not been determined for pulmonary function testing in this age group, finding an alternative measure to evaluate the efficacy of rhDNase therapy is crucial. In this study we used CXR and HRCT scores as objective measures of clinical response in this young patient population. The administration of rhDNase led to subjective improvement as reported by the parents, including decreased cough, increased activity level, improved appetite, and improved sleep. There were none of the complications of using rhDNase (alteration in voice, pharyngitis, and laryngitis) seen in older patients. ${ }^{7}$ The lack of adverse effects in our study population could reflect the small number of subjects and the short duration of the study. Wagener et al. ${ }^{8}$ also did not observe adverse effects in this age group.

There was improvement in both the CXR and the HRCT scores as compared with baseline values in the rhDNase group (Figs. 1, 2), while there was a worsening of both scores in the placebo group. The 


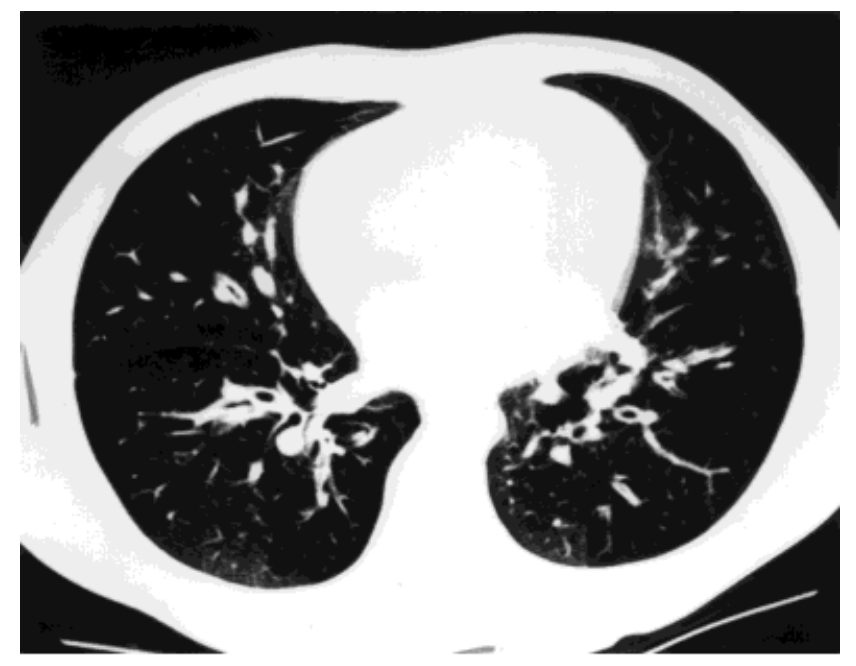

A

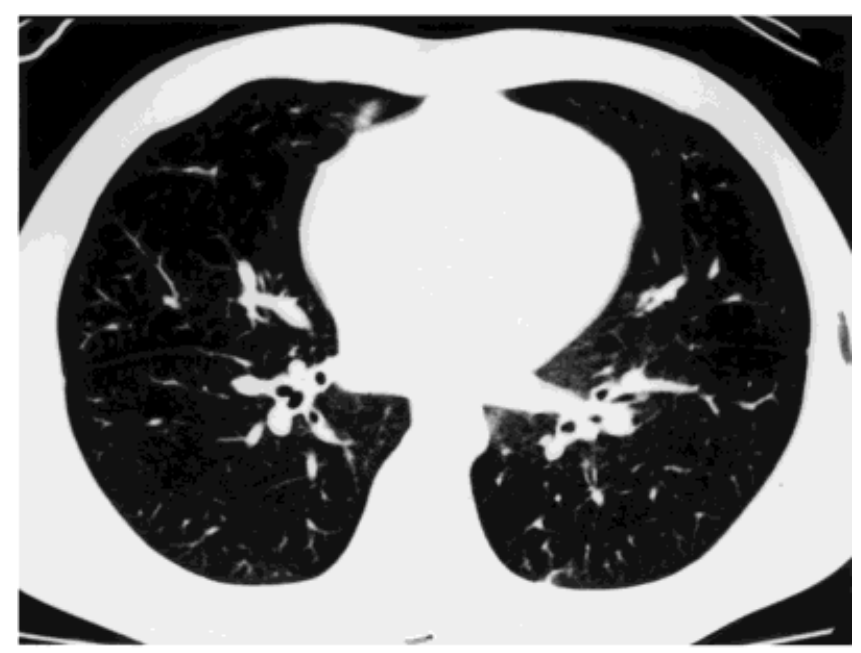

B

Fig. 1. High-resolution CT scan of subject (age 4.5 years) who received rhDNase. A: HRCT at baseline shows bronchial wall thickening and inhomogeneity of aeration of lower lobe (ground-glass appearance). B: HRCT at end of study showed improvement in all categories listed above.

interobserver coefficient of variation for CXR scores was excellent $(3.1 \%)$. The higher variation in HRCT scores $(49.1 \%)$ probably reflects the small range of the scoring system and the small number of patients. The current CT technology allowed us to perform the imaging procedure without sedation. This resulted in some motion artifact, especially in patients younger than 2.2 years. Performing the HRCT without sedation decreased the risk of the procedure, made it more acceptable to the parents, and decreased cost. The radiation dosage was less than conventional chest CT with our technique, as we acquired a limited number of slices (five) with a low $\mathrm{mA}$ and scan time. We estimated

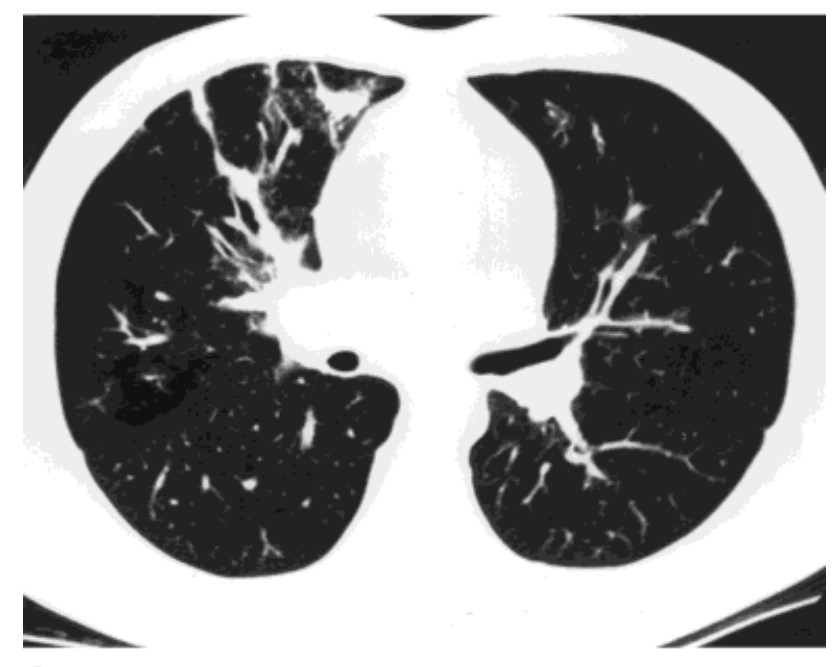

A

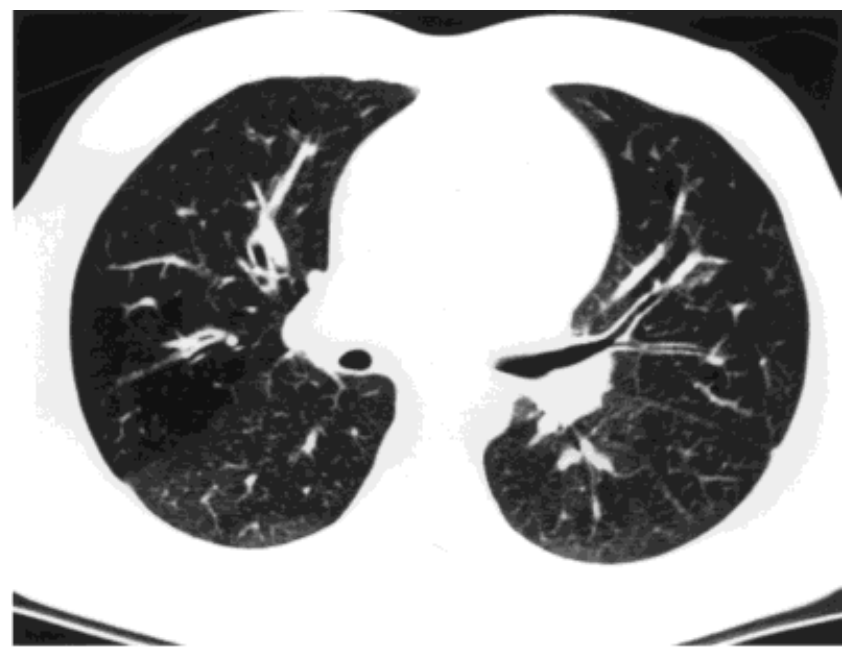

B

Fig. 2. HRCT scan of same patient in Figure 1, at different level. A: HRCT at baseline shows RML interlobular septal thickening, peribronchial thickening, and atelectasis. B: HRCT at end of study shows improvement in all categories listed above. CXR for that patient (not shown) revealed no significant changes in Brasfield score between baseline and at completion of study.

the integral dose to the thorax to be about a mean dose of $50 \mathrm{mRem}$, as based on Santamaria et al. ${ }^{20}$ since the mAs in this study were one-tenth of the technique in that reference. The CT will give peaks and valleys of radiation to the thorax. The CXR in our institution gives a maximum skin external dose of 30-50 mrem. The integral dose of the two procedures should be nearly equal, even though the dose is delivered in a different manner by the two procedures.

There was significant correlation between observers for both CXR and HRCT scores. This was consistent with the findings of Santamaria et al. ${ }^{20}$ There was no significant correlation between mean CXR and mean HRCT 
scores. This is likely related to the high variability in HRCT scores. It could also relate to the difference in sensitivities of CXR and HRCT scores.

There were significant correlations between most subscores and total HRCT scores, except for the number of bullae and collapse. These two subscores were not observed in this patient population with relatively mild $\mathrm{CF}$ disease. The subscores that correlated best with the total HRCT score were bronchiectasis, peribronchial thickening, mucus plugging, and air trapping.

Comparative analysis of the two patient groups revealed a statistically significant difference in HRCT scores. The CXR score also showed improvement in the rhDNase group and worsening in the placebo group. However, these differences did not reach statistical significance, probably due to the small sample size and the lower sensitivity of CXR vs. HRCT in the evaluation of early CF lung disease. These findings would support the hypothesis that in mild CF, the use of HRCT is more sensitive and likelier to detect differences between study groups, and should be considered in evaluating different therapeutic interventions in this patient population. The use of HRCT as described here provides radiation doses similar to those of CXR. However, the information provided by HRCT is far superior and gives higher sensitivity than CXR.

This preliminary study suggests that aerosolized rhDNase is a safe and effective agent in $\mathrm{CF}$ patients younger than 5 years. A long-term study in a larger patient population would be of interest to further evaluate the benefits of this therapeutic modality to slow the progression of CF lung disease. HRCT can be used to objectively assess the severity of CF lung changes in preschool children who cannot perform pulmonary function tests. HRCT appears to be a more sensitive study than CXR in evaluating this patient population.

\section{ACKNOWLEDGMENTS}

We thank Dr. Kimberly A. Garver for scoring the HRCT, Ramy Kurdi for preparing the manuscript, and Dr. Bruce Brock and Ron Shore for the statistical analyses.

\section{REFERENCES}

1. Boat TF. Cystic fibrosis. In: Murray JF, Nadel JA, editors. Textbook of respiratory medicine. Volume 1. Philadelphia: W.B. Saunders; 1988. p 1126-1152.

2. Lethem MJ, James SL, Marriot C. The role of mucous glycoproteins in the rheologic properties of cystic fibrosis sputum. Am Rev Respir Dis 1990;142:1053-1058.

3. Kirchner KK, Wagener JS, Khan TZ, Copenhaver SC, Acurso FJ. Increased DNA levels in bronchoalveolar lavage fluid obtained from infants with cystic fibrosis. Am J Respir Crit Care Med 1996;154:1426-1429.

4. Shak S, Capon DJ, Hellmiss R, Marsters SA, Baker CL. Recombinant human DNase 1 reduces the viscosity of cystic fibrosis sputum. Proc Natl Acad Sci USA 1990;87:9188-9192.

5. Ranasinha C, Assoufi B, Shak S, Christiansen D, Fuchs H, Empey D, Geddes D, Hudson M. Efficacy and safety of short-term administration of aerosolized recombinant human DNase in adults with stable stage cystic fibrosis. Lancet 1993;342:199202.

6. DNase Clinical Research Consortium. Efficacy and safety of aerosolized recombinant human DNase in patients with cystic fibrosis. Clin Res 1992;40:3184.

7. Fuchs HJ, Borowitz DS, Christiansen DH, Morris EM, Nash ML, Ramsey BW, Rosenstein BJ, Smith AL, Wohl ME, for the Pulmozyme Study Group. Effect of aerosolized recombinant human DNase on exacerbations of respiratory symptoms and on pulmonary function in patients with cystic fibrosis. N Engl J Med 1994;331:637-642.

8. Wagener JS, Rock MJ, McCubbin MM, Hamilton SD, Johnson CA, Ahrens RC. Aerosol delivery and safety of recombinant human deoxyribonuclease in young children with cystic fibrosis: a bronchoscopic study. J Pediatr 1998;133:486-491.

9. Cystic Fibrosis Foundation. Patient registry. 1997 annual data report. Bethesda, MD: Cystic Fibrosis Foundation; 1998.

10. Hansell DM, Strickland B. High resolution computed tomography in pulmonary cystic fibrosis. Br J Radiol 1989;62:1-5.

11. Nathanson I, Conboy K, Murphy S, Afshani E, Kuhn JP. Ultrafast computerized tomography of the chest in cystic fibrosis: a new scoring system. Pediatr Pulmonol 1991;11:81-86.

12. Bhalla $\mathrm{M}$, Turcios $\mathrm{N}$, Aponte $\mathrm{V}$, Jenkins $\mathrm{M}$, Leitman MJ, McCauley DI, Naidich DP. Cystic fibrosis: scoring system with thin-section CT. Radiology 1991;179:783-788.

13. Maffessanti M, Candusso M, Brizzi F, Piovesana F. Cystic fibrosis in children: HRCT findings and distribution of disease. J Thorac Imaging 1996;11:27-38.

14. Shah RM, Sexauer W, Ostrum BJ, Fiel SB, Friedman AC. High resolution $\mathrm{CT}$ in the acute exacerbation of cystic fibrosis: evaluation of acute findings, reversibility of those findings, and clinical correlation. AJR 1997;169:375-380.

15. Lynch DA, Brasch RC, Hardy KA, Webb RW. Pediatric pulmonary disease: assessment with high-resolution ultrafast CT. Radiology 1990;176:243-248.

16. Brody AS. Cystic fibrosis: when should high-resolution computed tomography of the chest be obtained? Pediatrics 1998;101: 1071.

17. Cystic Fibrosis Foundation Center Committee and Guidelines Subcommittee. The Cystic Fibrosis Foundation guidelines for patient services, evaluation, and monitoring in cystic fibrosis centers. Am J Dis Child 1990;144:1311-1312.

18. Mayo JR, Jackson SA, Miller NL. High-resolution CT of the chest: radiation dose. AJR 1993;160:479-481.

19. Goodsitt $M$, diagnostic radiologist physicist unpublished data, October 17, 1997.

20. Santamaria F, Grillo G, Guidi G, Rotondo A, Raia V, deRitis G, Sarnelli P, Caterino M, Greco L. Cystic fibrosis: when should high-resolution computed tomography of the chest be obtained? Pediatrics 1998;101:908-913.

21. Brasfield D, Hicks G, Seng-Jaw S, Tiller RE. The roentgenogram in cystic fibrosis: a new scoring system. Pediatrics 1979;63: $24-29$. 